\title{
Caracterización de los telebachilleratos comunitarios. El caso de Guanajuato
}

\section{The characterization of telebachilleratos comunitarios. The case in Guanajuato}

\section{Marcos Jacobo Estrada Ruiz* \\ SERgio Jacinto Alejo López**}

El artículo presenta resultados de una investigación más amplia cuyo objetivo es caracterizar a los telebachilleratos comunitarios en el estado de Guanajuato. Si bien estos no son nuevos en el país, sí lo son bajo la figura actual de telebachilleratos comunitarios. Esta opción educativa representa una estrategia de incremento de la cobertura para la educación media superior en la entidad, en particular en poblaciones rurales. Sin embargo, como subsistema relativamente nuevo, se desconoce su funcionamiento, características y condiciones de operación y desarrollo. Siguiendo la perspectiva de la evaluación sistemática, y a través del análisis documental y del trabajo de campo con los responsables de esta modalidad educativa en Guanajuato, este trabajo muestra una caracterización inicial que da cuenta de las condiciones que dieron origen a esta opción de educación media. Analiza las condiciones de desarrollo institucional y la relación conflictiva y estratégica con las telesecundarias y las comunidades. Como un punto importante de su caracterización, se destaca el ámbito comunitario, al cual se recurre para marcar su signo de distinción respecto a los demás subsistemas.

The article presents the results of a wide research having the aim to characterize Telebachilleratos Comunitarios in the state of Guanajuato. Despite having a long time operating in Mexico in other states, they have been recently created in Guanajuato. This educational opportunity is representing a strategy for increasing the offer of upper secondary education in rural communities around the state. In spite of the fact that Telebachilleratos Comunitarios are a novel subsystem in upper secondary education, its operation, characteristics and development are unknown. In this research, different means such as systematic assessment, documentary analysis and fieldwork were applied in order to obtain an initial characterization of Telebachilleratos that allows to be aware of the conditions that enabled the emergence of this subsystem. The conditions of the institutional development, and relationship issues among Telesecundarias, rural communities and Telebachilleratos are also analyzed in this study. A remarkable point shown as a result of Telebachilleratos characterization is that these educational centers work on-site among rural communities.

\section{Palabras clave:}

educación media superior, telebachilleratos comunitarios, cobertura educativa, evaluación sistemática

Keywords:

community
telebachilleratos,
upper secondary
education, systematic
evaluation,
educational coverage

Recibido: 8 de febrero de 2018. | Aceptado para su publicación: 24 de julio de 2018. Recuperado de: https://sinectica.iteso.mx/index.php/SINECTICA/article/view/841 DOI: 10.31391/S2007-7033(2018)0051-005

\footnotetext{
* Doctor en Educación. Profesor-investigador en la Universidad de Guanajuato. Miembro del Sistema Nacional de Investigadores (nivel 1). Sus líneas de investigación versan sobre formación ciudadana y procesos de construcción de ciudadanía; campo político y juventud; participación social en la educación; evaluación educativa; estudios sobre la educación media superior. Correo electrónico: estrada.ruiz.marcosj@gmail.com

**Doctor en Educación. Profesor-investigador en la Universidad de Guanajuato. Profesor con perfil deseable. Sus líneas de investigación versan sobre representaciones sociales; formación ciudadana y violencia; estudios sobre la educación media superior. Correo electrónico: jacintosergio@hotmail.com
} 


\section{INTRODUCCIÓN}

$\mathrm{E}$ ste trabajo forma parte de una investigación más amplia que ha tenido por objetivo caracterizar a los telebachilleratos comunitarios (TBC) de Guanajuato a través de la evaluación sistemática de tres de las dimensiones de la calidad educativa (De la Orden, 2007; INEE, 2009), en cuanto a pertinencia, relevancia y equidad del subsistema. En este artículo presentamos solo lo referente al trabajo documental y de campo realizado con los responsables estatales de los TBC, que dan cuenta de las dimensiones anteriores en sus aspectos generales, así como de una primera caracterización de estos en Guanajuato. Algunas de las preguntas que pretendemos contestar son: ¿cuáles son las valoraciones que los responsables de los TBC hacen sobre este tipo de opción educativa? ¿Cómo el TBC se ha convertido en una estrategia de incremento de la cobertura? ¿Cuáles son sus características en Guanajuato?

El caso de Guanajuato es en particular interesante al menos por dos razones: una es que la operación del subsistema de TBC le fue otorgada a una universidad de relativa reciente creación, la Universidad Virtual del Estado de Guanajuato (UVEG), y la otra es que constituye la segunda entidad en el país, solo después del Estado de México, que ha creado más TBC (354 y 520, respectivamente). En virtud de estas condiciones, consideramos que, del conocimiento profundo de este caso, se pueden desprender perspectivas de análisis para comprender otros procesos similares en el país.

La educación media superior en México ha venido enfrentando en los últimos años diversas problemáticas, las cuales cobraron mayor evidencia desde la Reforma Integral de la Educación Media Superior (RIEMS) en 2008, cuando se puso en el centro del debate a este nivel educativo; entre otros, destacan varios indicadores: los altos porcentajes de deserción, reprobación, lo referente a la calidad en los aprendizajes, y el trasfondo de la cobertura, que sigue asociada a las desventajas territoriales, las desigualdades sociales y las características sociodemográficas (Solís, 2018).

La cuestión de la cobertura obtuvo mayor importancia desde 2012, cuando se instauró el carácter obligatorio del nivel medio superior (Ramírez, 2015), y se puso como meta el ciclo escolar 2021-2022 para lograr la cobertura total. Ramírez considera que, de esos cambios, se derivaron tres desafíos: acelerar el crecimiento de la oferta en educación media superior (EMS), combatir el abandono escolar y mejorar de forma sustantiva la calidad de la oferta educativa. Justamente en lo referente al incremento de la cobertura, entre los motores del crecimiento de la EMS, además de los deseos de mayor escolaridad de los jóvenes y sus familias, se encuentra el aumento de la oferta institucional de este tipo de servicio educativo (Weiss, 2015). En este contexto, una de las estrategias que el Estado mexicano ha priorizado es la de crear nuevas opciones educativas, entre ellas el TBC (Weiss, 2017), subsistema en el que se concentra nuestro estudio.

\section{Algunos AnTECEDENTES DE LOS TBC}

Esta modalidad tiene antecedentes históricos en el sistema educativo nacional, en particular en los telebachilleratos iniciados en la década de los ochenta del siglo XX en diversas entidades del país (Espinoza, 2014), y parecen mantener parte de su sentido y lógica: estar destinados a ampliar la cobertura en la EMS y resolver el rezago educativo 
(Salazar, 2007), con lo cual se beneficia a comunidades dispersas de los estados y se fomenta el arraigo de los estudiantes a sus lugares de origen; a ello se agrega el uso de medios audiovisuales (Omaña, 2001).

No hay muchos estudios sobre el tema, particularmente a partir de lo que podemos denominar una nueva etapa de los telebachilleratos, ya en un contexto de obligatoriedad de la EMS y con la RIEMS iniciada en 2008. Aun así, los trabajos existentes muestran ciertas tendencias importantes, por ejemplo, en referencia a nuestro tema, el hecho de que existe una fuerte interrelación de las escuelas con las comunidades donde están ubicadas, es decir, una suerte de educación vinculada a la comunidad (Omaña, 2001). Asimismo, se ha encontrado una relación positiva entre la construcción de espacios comunitarios de intercambio entre autoridades y jóvenes, que lleva a que se amplíen las percepciones de estos más allá de las construidas en la escuela (Rosas, Santiago y Lara, 2015). No obstante lo anterior, también se ha presentado su contraparte: las percepciones negativas que tienen otros sectores de la población sobre estas modalidades educativas, al señalar que ofrecen una educación de menor calidad (Rosas, Santiago y Lara, 2015).

Los pocos estudios sobre este tema destacan diversas ventajas de esta modalidad que los propios actores consideran, como la aparente rapidez con la que se instalan e inician operaciones, el bajo costo con relación al alumnado que se atiende, además de que se trabaja en comunidades donde la construcción de un gran centro no es una opción (Salazar, 2007). Lo anterior habla del tipo de población al que está dirigido, así como su pertinencia y las condiciones de equidad que lo envuelven como opción educativa. De acuerdo con Salazar (2007), esta modalidad ha contribuido a atender el rezago educativo, pero trae aparejada su problemática inherente: la calidad.

El trabajo reciente de Weiss (2017a) puede considerarse pionero o al menos el primer estudio sobre lo que hemos denominado una nueva etapa de los telebachilleratos. Entre lo más destacable concerniente a nuestro tema, se muestra que hay estados donde existe un número apreciable de TBC que se han establecido en ámbitos urbanos, lo que varía por estados; por ejemplo, en Aguascalientes se explica por qué el TBC absorbió al telebachillerato estatal que ya existía. En el caso de Guanajuato, dicho estudio revela que la razón es la gran demanda de zonas urbanas periféricas que no están absorbiendo otros tipos de servicio; además, para algunos estados parece convenir que sus bachilleratos estatales se transformen y logren cierto financiamiento con un servicio como el TBC (Weiss, 2017a).

En Chihuahua y Guanajuato, los TBC se establecieron en lugares estratégicos donde convergen las comunidades $y$, de esta forma, se amplía su alcance. La situación de Chihuahua es peculiar porque los TBC, en su gran mayoría, han buscado tener sus propias instalaciones a fin de evitar conflictos con las telesecundarias y por cuestiones de seguridad (Weiss, 2017).

En cuanto a las dificultades de inicio de los TBC, la problemática más recurrente es la aceptación de las poblaciones de las localidades, en especial por la incertidumbre de los padres sobre si poseen reconocimiento oficial. La labor inicial de difusión y conocimiento parecen haberla hecho los profesores, casa por casa, convenciendo a los padres y a los jóvenes (Weiss, 2017a). 
Algunos elementos que aportan a la caracterización los ofrece también el trabajo de Weiss (2017a, 2017), quien menciona que el salario de los docentes es cubierto en su totalidad por el gobierno federal durante el primer año; en el segundo, el esquema cambia a $50 \%$ federal y otro $50 \%$ el gobierno estatal, a excepción de Chihuahua, donde el Gobierno del Estado cubre el salario por completo.

En virtud de las características de los docentes del TBC, se le denomina como "un subsistema joven con docentes jóvenes" (Weiss, 2017a, p. 37). En las entidades exploradas, los profesores son jóvenes profesionales que tienen poco tiempo de haber egresado de distintas licenciaturas e ingenierías variadas. El proceso de contratación es diverso y depende de cada entidad; por ejemplo, en el Estado de México se reportó que se contrataba a muchos recomendados e influyentes, aunque también se aclara que se encuentran en un proceso de transición, al estar permeado por el Servicio Profesional Docente (Weiss, 2017).

\section{DESDE LA EVALUACIÓN SISTEMÁTICA}

Con base en la perspectiva de Stufflebeam y Shinkfield (1987), la evaluación debe estar orientada hacia el perfeccionamiento, y los informes evaluativos tendrían que ayudar a los responsables a tener una mejor comprensión del fenómeno evaluado y que esto contribuya a perfeccionar un determinado proceso, al brindar información útil para la toma de decisiones. En otras palabras, la evaluación en esta postura implica tener claros tres propósitos evaluativos: servir de guía para la toma de decisiones; proporcionar datos para la responsabilidad; y promover la comprensión de los fenómenos implicados (Stufflebeam y Shinkfield, 1987). Así, la evaluación es presentada como un proceso más que una prueba, y se fundamenta en tres etapas claras, que son identificar, obtener y proporcionar información.

Si el método CIPP (context, input, process, product) es llevado hasta el final, como dice Stufflebeam y Shinkfield (1987), se pueden plantear diversas preguntas que corresponden a cada uno de los momentos evaluativos en los que se fundamenta. Los momentos que dan nombre al método son: evaluación de contexto, de entrada, del proceso y del producto.

En la evaluación del contexto se identifica a la población objeto del estudio o beneficiaria de las acciones, y se determina la coherencia de las necesidades de la población y los objetivos planteados, así como de las estrategias proyectadas y, en general, el marco institucional desde el cual se emprenden los procesos.

La evaluación de entrada identifica la capacidad del sistema, de la institución o de los actores que llevan a cabo el proceso formativo o de intervención, así como de las estrategias planificadas y los presupuestos (teóricos y, en algunos casos, económicos). Por su parte, la evaluación del proceso, que es tradicionalmente concebida como la acción de identificar o pronosticar los defectos de la planeación, en nuestro trabajo se refiere más a las estrategias formativas y a la descripción y análisis del desarrollo de las intervenciones institucionales.

Por último, la evaluación del producto es la recopilación de las descripciones y los juicios de los actores sobre su proceso, en este caso en el TBC, relacionados con los objetivos y las metas propuestas. Fundamentalmente, es la conjunción de la 
evaluación del contexto, de la entrada, del proceso y los juicios de los actores, que, en conjunto, permiten determinar el valor y el mérito de las actividades y acciones llevadas a cabo en las estrategias formativas y de intervención.

Cada etapa anterior, si bien no es lineal, sí requiere un momento específico para su realización. En nuestro estudio, las dos primeras se abordan desde el análisis de los documentos y toda la planeación con la que ya se puede contar y que proviene de las instituciones responsables, como la Secretaría de Educación Pública y la Subsecretaría de Educación Media Superior (SEP-SEMS), además de las consultas con los propios responsables de los TBC en Guanajuato, de la revisión de los recursos humanos y materiales para enfrentar las acciones, de la viabilidad y coherencia de lo planteado. Es, en general, una evaluación sobre el diseño, el contenido y las estrategias formativas planeadas en esta modalidad educativa, así como la comprensión de los conceptos básicos que la guían. Las dos siguientes -evaluación del proceso y del producto- se atienden desde el trabajo de campo, el contraste de lo planeado con el desarrollo e implementación, y la valoración de los actores.

Estos cuatro momentos del método CIPP nos proporcionan datos referentes a la relevancia, pertinencia y equidad, al indicarnos, por ejemplo, los contenidos y propuestas curriculares de esta clase de bachilleratos; la perspectiva de los responsables de los TBC, desde la propuesta de creación y funcionamiento hasta el tratamiento concreto en aula $\mathrm{y}$ en los procesos formativos en general.

\section{Metodología}

En la investigación de la cual se desprende este artículo, construimos una metodología cualitativa e instrumentos de investigación guiados por la perspectiva y enfoque asumido, es decir, el modelo CIPP de Stufflebeam y Schinkfield (1987). La metodología cualitativa la entendemos como aquella que produce hallazgos sin recurrir necesariamente a estrategias de cuantificación; más bien se llega a ellos por medio de procesos interpretativos (Strauss y Corbin, 2002).

En este artículo nos enfocamos solo al trabajo de campo y a la parte metodológica que concierne a la caracterización de los TBC de Guanajuato, construida con la revisión documental de los textos base provenientes de la SEMS y de las entrevistas en profundidad realizadas en la UVEG a los responsables de los TBC en el estado. La entrevista la concebimos como técnica que ayuda a entender el mundo desde la perspectiva del entrevistado y contribuye a desmenuzar los significados de sus experiencias (Álvarez, 2003). Realizamos entrevistas en profundidad que suponen superan la versión oral de una encuesta rápida (Angrosino, 2012); ya con el guion construido, buscamos los significados de los actores.

Las entrevistas se aplicaron a actores responsables de las áreas encargadas, dos integrantes de la Dirección de Educación Media Superior y tres miembros del área de vinculación de la región norte y sur. A todos se les pidió autorización para grabar las entrevistas y usar los datos con la certeza de que su identidad sería salvaguardada; tuvieron una duración aproximada de poco más de una hora; con uno de los actores fueron dos sesiones con el mismo tiempo de duración. Las entrevistas fueron transcritas y sometidas al proceso formal de reducción de datos y organización 
según nuestros intereses y con base en el modelo CIPP. La lectura de los documentos del TBC fue guiada por su ubicación en el marco del mismo modelo.

A partir de los objetivos del modelo CIPP, construimos los guiones de entrevista y los instrumentos de su aplicación, los cuales nos aportaron datos para la caracterización (ver tabla 1).

Tabla 1. Momentos del modelo CIPP y dimensiones exploradas en las entrevistas

\begin{tabular}{|c|c|c|c|}
\hline Evaluación del contexto & Evaluación de entrada & Evaluación del proceso & Evaluación del producto \\
\hline $\begin{array}{c}\text { Comprensión y definición } \\
\text { del objetivo del TBC } \\
\text { Tipo de población al que } \\
\text { va dirigido el TBC } \\
\text { Formación que requiere } \\
\text { la población a la que va } \\
\text { dirigido el TBC. } \\
\text { Maneras en que el TBC } \\
\text { responde a las necesida- } \\
\text { des de formación de esta } \\
\text { población } \\
\text { Estrategias y modalidad } \\
\text { de trabajo en el TBC } \\
\text { Proceso de asignación de } \\
\text { los TBC a la UVEG } \\
\text { Responsabilidades de } \\
\text { las instancias federales y } \\
\text { estatales } \\
\text { Tipos de apoyo o segui- } \\
\text { miento que se le da a cada } \\
\text { escuela }\end{array}$ & $\begin{array}{l}\text { Condiciones para la crea- } \\
\text { ción de un TBC } \\
\text { Gestiones requeridas para } \\
\text { la apertura de los TBC } \\
\text { Apoyo que brindan las } \\
\text { distintas instancias } \\
\text { Aportaciones de la comuni- } \\
\text { dad y otras entidades } \\
\text { Proceso de gestión, contra- } \\
\text { tación de profesores } \\
\text { Perfil docente que se busca } \\
\text { Formación y actualización } \\
\text { de docentes en el TBC } \\
\text { Seguimiento que se da a los } \\
\text { resultados de evaluaciones } \\
\text { docentes } \\
\text { Principales problemáticas } \\
\text { que enfrentan los TBC }\end{array}$ & $\begin{array}{l}\text { Tramos de responsabilidad } \\
\text { y decisiones en los TBC } \\
\text { Organización y sistematiza- } \\
\text { ción de la información que } \\
\text { se recopila de cada TBC } \\
\text { Cómo se prioriza y se orga- } \\
\text { nizan las acciones } \\
\text { Materiales e insumos que } \\
\text { se brindan a los directivos y } \\
\text { docentes } \\
\text { Acreditaciones o evalua- } \\
\text { ciones externas al sistema } \\
\text { nacional de bachillerato, } \\
\text { padrón de buena calidad } \\
\text { Programas alternos o com- } \\
\text { plementarios } \\
\text { Seguimiento de la trayecto- } \\
\text { ria de los estudiantes }\end{array}$ & $\begin{array}{c}\text { Valoración general que como } \\
\text { responsables tienen del TBC } \\
\text { Cómo se mide el cumplimiento } \\
\text { de los objetivos y las metas } \\
\text { propuestas } \\
\text { Áreas de impacto de los TBC } \\
\text { Seguimiento de egresados } \\
\text { Principales retos y prospectiva } \\
\text { del TBC } \\
\text { Conocimiento de la transición } \\
\text { escolar o empleabilidad } \\
\text { Factores que influyen en el } \\
\text { abandono escolar, reprobación, } \\
\text { becas, convivencia, retención, } \\
\text { etcétera }\end{array}$ \\
\hline
\end{tabular}

La información obtenida fue suficiente para caracterizar los TBC desde la perspectiva de sus responsables a nivel central, quienes dan seguimiento administrativo y en campo a estos telebachilleratos.

\section{CARACTERIZACión DE LOS TBC CON BASE EN LAS EVALUACIONES DE CONTEXTO Y DE ENTRADA}

El TBC corresponde a la educación media superior y es un modelo que, a diferencia de otros subsistemas similares, ofrece educación escolarizada. Su creación se inscribe dentro de las estrategias del Estado mexicano para incrementar la cobertura a partir de la obligatoriedad de la educación media decretada en 2012. Después de un periodo piloto durante 2013 y de un proceso de "evaluación y ajustes" (SEMS, 2014), inició formalmente en el ciclo escolar 2014-2015.

El diagnóstico que fundamentó la propuesta de esta opción educativa era que una fracción importante de la población que no tenía acceso a la EMS se ubicaba en localidades pequeñas, y dado su tamaño, se argumentaba que no era factible tener un plan- 
tel en cada una de ellas. Por mandato constitucional y también por equidad (SEMS, 2016), se requería extender la cobertura y cubrir la demanda de esta población. En el estado de Guanajuato, la contribución y el cumplimiento de lo anterior se refleja en el indicador de tasa neta de cobertura, cuya evolución de 2010 a 2015 ha sido de más de diez puntos. En el ciclo escolar 2010-2011, el estado reportó 42.7 de cobertura, tuvo un crecimiento de dos puntos por ciclo y su mayor extensión la mostró en el ciclo escolar 2015-2016, con 53.9; esto, quizá, se asocie al crecimiento importante que la opción de TBC ha tenido en la entidad (INEE, 2012, 2013, 2014, 2015, 2016, 2017).

En su fase piloto a nivel nacional, el TBC inició en 2013 con 253 planteles, en 2014 ascendió a 1,497, en 2015 llegó a 2,918, y en el ciclo escolar 2017-2018, a 3,318 planteles; hasta esos años registró una matrícula de 131,000 estudiantes con 9,700 docentes (SEMS, 2018). De acuerdo con lo que consideramos la política base del TBC, argumentamos que con este se concreta el cumplimiento constitucional de cobertura, en particular en poblaciones pequeñas de hasta 2,500 habitantes.

Los objetivos del servicio educativo de TBC son dos:

Ampliar la cobertura de educación EMS a la población que por diversas razones no puede acceder a un plantel convencional [...] [y] contribuir a elevar el nivel educativo de la población, junto con los demás servicios existentes para atender en primera instancia a las y los jóvenes en edad típica de cursar el bachillerato (SEMS, 2016, p. 7).

Además, se añade que la población objetivo son los egresados de las secundarias de las localidades rurales que opten por dicho servicio. Este modelo está diseñado para atender a comunidades que tengan entre 12 y 30 alumnos de primer ingreso. En su documento base se enuncia que se tiene que hacer uso de todos los recursos disponibles en la localidad y en las instalaciones existentes (SEMS, 2014). La propuesta de los TBC es clara: "No habrá inversión en infraestructura” (SEMS, 2014).

La Subsecretaría de Educación Media Superior (SEMS) informa que existen en el país 18,200 telesecundarias, de las cuales 42\% no cuentan con una opción de EMS en un radio de cinco kilómetros. Esto es una muestra de la necesidad educativa que existe en el país, y con una población joven en particular que se encuentra en localidades con estas características: rurales y alejadas de los centros urbanos.

Las definiciones básicas que son aportadas por la SEMS ayudan a formular una primera caracterización general de los TBC: se establecen de preferencia en las telesecundarias o en los espacios que disponga la comunidad; es una modalidad escolarizada presencial que sigue el plan de estudios del bachillerato general; su plantilla docente es mínima, es decir, tres profesores; se apoya en materiales impresos y audiovisuales y la duración mínima para cursarlo es de tres años y máxima de cinco (SEMS, 2016).

De acuerdo con lo plasmado en la RIEMS, el plan de estudios del TBC está organizado por tres componentes de formación: básico, propedéutico y profesional, con la diferencia de que a los TBC se les agrega en la formación propedéutica las asignaturas de Derecho, Probabilidad y estadística, Ciencias de la comunicación y Ciencias de la salud, y en la formación profesional la capacitación que se ofrece es Desarrollo comunitario.

En cada plantel de TBC hay al menos tres docentes, que responden a un perfil disciplinario de cada área considerada: matemáticas y ciencias experimentales, ciencias sociales y humanidades, y comunicación. Uno de estos docentes funge como 
responsable de centro. El perfil docente considerado para este tipo de modelo es, en general, el que se consigna en el documento base del TBC; entre otras características y capacidades, debe mostrar dominio de la didáctica y del proceso enseñanza-aprendizaje para promover competencias, desarrollo de proyectos formativos, conocimiento del área que impartirá, sensibilidad para la identificación de necesidades de atención a estudiantes, manejo de estrategias frente a grupo, y sentido de responsabilidad y profesionalismo (SEMS, 2016). Deberá cumplir, además, con las competencias establecidas en el Acuerdo Intersecretarial 447 (Diario Oficial de la Federación, 2008), que precisa los principales atributos del perfil docente de EMS en modalidad escolarizada.

El trabajar por áreas disciplinares y no por asignaturas se debió a la necesidad de buscar la interdisciplinariedad para el tratamiento de los contenidos en el desarrollo de proyectos formativos (SEMS, 2016). Aunque lo anterior en el diseño parece el camino lógico y esperado, hemos observado que en la implementación para el estado de Guanajuato hay más bien un tratamiento basado en la lógica de materias, y que el diseño o la expectativa anterior ha sido difícil de cumplirse.

Por las particularidades de esta opción educativa, se han elaborado materiales didácticos ex profeso: a los estudiantes se les otorgan libros de asignaturas y a los profesores, guías para su ejercicio en los TBC. También se ofrece un curso propedéutico, que es una suerte de diagnóstico para docentes y estudiantes, en el que muestran su dominio en las habilidades matemáticas y de comprensión lectora; esto se aplica a los estudiantes de nuevo ingreso. Por último, las series audiovisuales consisten en 195 programas que se transmiten por la Red Edusat o son reproducidos en DVD (SEMS, 2016).

En cuanto a la organización del TBC, el número de alumnos para atender en el primer ingreso fue de entre 12 y 30, además de que se tendrían que usar todos los recursos disponibles de la localidad en la que se establecieran. Su coordinación estaría a cargo de quienes se designaran en cada entidad. En el caso de Guanajuato, la UVEG fue la responsable.

La estructura organizativa de los TBC es relativamente sencilla: de inicio, está el responsable estatal, que entra en relación con el responsable del centro, y le siguen los otros dos docentes. Aunque parece que en cada estado puede tomar una lógica distinta o más amplia, pues en el documento base se comenta que, dependiendo de los recursos y consideraciones estatales, se puede incorporar la figura del supervisor. En Guanajuato, además de la estructura anterior, sí aparecen los supervisores, organizados en regiones.

Aunque la descripción de las funciones de los docentes y los responsables de centro son diferentes, ambas coinciden en el perfil profesional y laboral: el docente responsable deberá tener experiencia docente de por lo menos un año en EMS y el docente, "un año de experiencia en educación". La diferencia radica en su tipo de contratación: el responsable del centro se contrata por $3 / 4$ de tiempo, es decir, 30 horas semana-mes, de las cuales 20 son para docencia y 10 para atender cuestiones de la dirección, mientras que los docentes, por medio tiempo, 20 horas semana-mes (SEMS, 2016, pp. 29-30).

La forma de trabajar en el TBC marca diferencia respecto al bachillerato general. Las actividades se tienen que preparar de manera colegiada desde el área disciplinaria, no de cada asignatura; por lo mismo, se debe trabajar con base en un proyecto 
formativo, una vez que se han identificado los problemas de la comunidad y, en conjunto con los estudiantes, se determinan los proyectos. Se tiene que elaborar por lo menos un proyecto formativo interdisciplinario en cada semestre (SEMS, 2016). El documento base define proyecto formativo como una "serie de actividades, articuladas y planeadas que se originan a partir de una problemática o situación de interés de los estudiantes y de la comunidad, que es propicia para el desarrollo de habilidades y la adquisición de conocimientos" (SEMS, 2016, p. 33).

A su vez, el documento base del bachillerato general, al cual corresponde o se circunscribe el TBC, muestra algunos elementos que ayudan a entender y valorar el hecho de que sean parte de este tipo de bachillerato. En primer lugar, en el sentido otorgado a la EMS, considerada como estratégica para el desarrollo del país, además de promover la participación creativa de las nuevas generaciones en la economía, el trabajo y la sociedad. Para lo anterior, la EMS está integrada por dos opciones educativas: una propedéutica y otra bivalente. La primera, a la cual responde el TBC, prepara a los jóvenes para el estudio de las diferentes disciplinas científicas tecnológicas y humanistas, además de que proporciona una cultura general con la finalidad de que los egresados se incorporen a las instituciones de educación superior y al sector productivo (SEMS, 2016).

Sin embargo, en lo que se refiere al TBC, no significa que no existen contenidos y formación relacionada con una actividad profesional o para vincularse al campo laboral. De manera explícita, se concibe la materia de desarrollo comunitario con esta función, es decir, como capacitación para el trabajo (SEMS, 2014a, 2017). A partir del tercer semestre y hasta el sexto, se desarrolla esa materia en los módulos siguientes: en el tercer semestre, Significados del desarrollo comunitario, elementos de una comunidad; en el cuatro, Elementos para elaborar un diagnóstico comunitario; en el quinto, Elaborar un proyecto de desarrollo comunitario e instrumentarlo; por último, en el sexto semestre, Evaluar los alcances del proyecto de desarrollo comunitario y elaborar un plan de seguimiento y continuidad del proyecto (SEMS, 2014 b). Uno de los objetivos que más llama la atención de esta materia es el de contribuir al arraigo y compromiso de quienes egresan de los TBC.

\section{CARACTERIZACIÓn DESDE LA EVALUACIÓN DEL PROCESO Y DEL PRODUCTO}

Hay dos tipos de control o seguimiento que en la UVEG se realizan sobre los TBC, uno más administrativo y otro más académico. En el área de vinculación se establece una coordinación encargada de darle seguimiento a los TBC, organizados en tres regiones, y cada una de estas cuenta con cinco supervisores, los cuales, sin embargo, no operan desde la lógica de otros subsistemas o niveles educativos, que es más una cuestión administrativa, aunque impacta en lo educativo; su trabajo es previo a la labor académica que viene después. Así lo señala una de las responsables: "Es llevar la promoción y realizar la captación de los alumnos [...] que en este caso pues es desde hablar con los padres de familia, hacer campaña de difusión que va desde perifoneo, pega de papeles, volanteo" (EAV). Esto, de inicio, nos sirve para conocer la manera en que se difundió el TBC y fue captando estudiantes, un proceso que ahora, relatan, ya no llevan a cabo en la misma medida, porque han logrado cierta presencia en las comunidades. 
La importancia del área de vinculación radica en que es la instancia que inicia el proceso de gestión de la creación de un TBC y se mantiene presente después de esto, pues constituye, según los responsables de ella, los ojos de las demás áreas. Aunque quizá no sea su función principal, es cierto que, además, se encarga de ejercer un tipo de supervisión del servicio educativo en cada centro escolar.

En general, el área de vinculación consiste en un grupo de trabajo que

está conformado por quince supervisores a nivel estatal. Son tres coordinadores regionales, ellos están en campo viendo que los centros estén operando [...], que tengan la infraestructura necesaria para prestar el servicio, que estén presentándose los docentes, que estén también presentándose los alumnos y que prácticamente todo se esté dando (EAV).

La comunicación con cada centro se da a través de los maestros, y representa un reto que ha tenido que resolverse desde el inicio, en particular por las zonas donde se encuentran las escuelas, no solo de acceso, sino de conexión a internet. La manera en que se organiza el trabajo de la coordinación de vinculación y acompañamiento a los TBC logra superar las distancias en las que se localizan los supervisores, y lo hace con reuniones mensuales en la sede de la UVEG en el municipio de Irapuato, es decir, los quince supervisores deben trasladarse de los distintos municipios donde se ubican.

La manera en que se logra, desde las escuelas, la relación con los supervisores y las tres coordinaciones regionales es a través de la cadena o línea de mando; es decir, una incidencia o reporte desde cada escuela debe dirigirse, en primer lugar, a su supervisor, sigue la cadena al coordinador que corresponda; también, como nos comentaron los responsables, tienen un sistema de incidencias en línea que permite elaborar un reporte, en el cual quedan asentados el día y la hora en que se levantó y el área al que va dirigido, incluyendo el tiempo en el que se le dio solución o se atendió. Es un sistema automatizado que ha servido para salvar las distancias y la comunicación desde las zonas alejadas, y por la alta demanda de información de los docentes. Las incidencias o reportes más recurrentes de las escuelas se refieren a las aulas, las cuestiones de infraestructura y la convivencia con las telesecundarias.

La presencia de la UVEG a nivel estatal explica por qué se le asignó la operación del TBC; aparte de su corta existencia como universidad virtual (diez años), su visibilidad en cada municipio del estado a través de los centros de acceso educativo (CAE) ha sido el germen no solo de su funcionamiento, sino de donde han salido los recursos humanos que dieron origen y atienden actualmente al TBC.

\section{La perspectiva de la Dirección de Educación Media de la UVEG}

Además de la Dirección de Vinculación, la otra parte fuerte que atiende a los TBC es la Dirección de Educación Media, aunque más en el aspecto académico. Sin embargo, las actividades que los responsables mencionan están caracterizadas como de administración escolar; por ejemplo, citan entre las funciones el control administrativo-escolar, la gestión de elementos como constancias, la distinta documentación que se requiere en los procesos educativos, las becas, etcétera. En concreto, la parte fuerte de la actividad es el control escolar; ante la existencia de los TBC en toda la entidad y su ubicación en las zonas más remotas del estado, el dar seguimiento a esas labores es todo un reto, el cual ha sido parcialmente resuelto al tomar ventaja 
de lo que mencionan como el "sacar provecho a las tecnologías de la información", en lo cual, por la misma naturaleza de la UVEG, tienen conocimiento y experiencia.

Así, desde las oficinas centrales en el municipio de Irapuato se controla y concentra prácticamente todo, combinando lo virtual y lo presencial: "Nos envían la información, nos citamos, hacemos videoconferencias, etcétera, y ahora también tenemos otro ámbito que se llama reuniones regionales [...] y cada dos meses estamos reuniéndonos con los profesores por zonas" (EDEM).

En este caso, además de la comunicación virtual y presencial, se tiene cierta estructura de seguimiento y funcionamiento que permite saber lo que acontece en cada plantel y cómo esto llega hasta la sede donde se toman decisiones. En la Dirección de Educación Media de la UVEG (al igual que en el área de vinculación), se considera que el primer contacto es el promotor que existe en cada CAE de los municipios; son, por decirlo así, sus ojos permanentes, quienes establecen visitas y observan el contexto básico donde ya opera o puede funcionar un nuevo TBC. Después viene el área de vinculación, con la cual establecen una relación al mismo nivel:

Nos sentamos a la mesa y vamos determinando como situaciones emergentes que se vayan dando, casi siempre es por comunicación directa, cuando al promotor le surge alguna inquietud, el promotor lo externa a su vinculador que, digamos es su supervisor y su supervisor nos llama cuando se pondere que es una situación académica y se atiende lo más pronto posible (EDEM).

En la atención a las necesidades que se van presentando, la estructura de la organización de la UVEG dirige a cada área lo que le corresponde, y en campo, en las escuelas, esta distinción parecen también tenerla clara.

\section{La creación de los TBC}

Una de las preguntas principales en una entidad con el mayor número de TBC en el país, solo después del Estado de México, es cómo se crean estos. Parte de la respuesta proviene de la variedad de orígenes de las gestiones que se pueden hacer. Los responsables de los TBC comentan que puede venir de las autoridades municipales, de la UVEG por ejemplo, y pasan por las delegaciones de educación que operan en el estado. Con estas, la UVEG entra en relación primaria para la creación de un nuevo centro educativo: "Nosotros nos sentamos previamente con ellos para determinar cuáles son las comunidades propuestas, pero también filtrar cuáles de ellas son las que cumplen con los lineamientos que establece la federación" (EAV).

Al mismo tiempo, al trabajo de escritorio le acompañan las estrategias en campo que el área de planeación de la UVEG realiza con el estudio de factibilidad, además de una visita in situ cuya función es verificar que los datos de las cifras previas coincidan con lo que sucede en las comunidades y escuelas existentes y en la población en general. Lo anterior tiene la finalidad de garantizar que la apertura de un nuevo centro cuente con las instalaciones adecuadas y sean acordes con la normativa, de tal manera que la UVEG y la Secretaría de Educación de la entidad estén en condiciones de formular una mejor propuesta. 
La propia necesidad histórica y la exigencia actual del incremento de la cobertura es, sin embargo, la que dirige la apertura y la relativa facilidad y rapidez con la que se crean los TBC. Asimismo, la gestión se inicia desde la UVEG para que la comunicación funcione y se hagan alianzas en las áreas de educación básica, pues hay que recordar que los TBC se establecen usando las instalaciones de las telesecundarias. Vale la pena mencionar que es en estas últimas donde se hace evidente la necesidad; por eso, quizá, comparten, en la mayoría de las ocasiones, el interés por la apertura de nuevos centros: "Si ellas [las telesecundarias] no nos dieran la apertura, porque están muy preocupadas en que se pueda dar esta posibilidad de estudio en esas comunidades, no habría posibilidad de que los muchachos continuaran estudiando" (EAV).

A grandes rasgos, lo anterior sería el esquema que se sigue para la creación de un TBC en Guanajuato, y muestra, en especial desde la evaluación del proceso, las estrategias institucionales para la expansión del subsistema. Una vez que se aprueba y tiene que iniciar operaciones un nuevo centro, surge la segunda etapa, en la cual es importante la experiencia de la UVEG y los recursos que posee en cada municipio del estado. Se inicia con la difusión de una nueva opción educativa en las comunidades, por medio del perifoneo, volanteo, carteles, y, en particular, con la información que de persona en persona hacen los mismos habitantes de las comunidades donde impacta un TBC. Esta última parte se da cuando ya ha iniciado el centro educativo: "Si el centro está operando bien, por sí solo vende, no necesita un solo volante, un solo flyer para poder vender, los muchachos se empiezan a interesar, digamos esa es nuestra principal fuente de promoción, la gente" (EDV).

Siguiendo el discurso de los responsables de las áreas analizadas de la UVEG, en la tabla 2 se muestra la lógica para la creación de un nuevo TBC.

Tabla 2. Esquema para la creación de un TBC

\begin{tabular}{|c|}
\hline "La federación nos manda cuáles son las bases para la apertura" (EDV) \\
\hline $\begin{array}{c}\text { "Empezamos a socializar esto con delegados, con presidentes municipales, con autoridades educativas, para } \\
\text { conocer cuáles son las necesidades en los municipios y en las comunidades en donde podríamos llegar" (EDV) }\end{array}$ \\
\hline $\begin{array}{c}\text { "Que pudiéramos tener a los alumnos, al menos doce para comenzar el servicio y empezamos a hacer todas } \\
\text { las gestiones en la comunidad" (EDV) }\end{array}$ \\
$\begin{array}{c}\text { "Nos dirigimos directamente a las comunidades, hablamos con los padres de familia, hablamos con el de- } \\
\text { legado de la comunidad que es para nosotros un gran aliado y entonces comenzamos a hacer las gestiones, } \\
\text { comenzamos a hacer la vinculación" (EDV) }\end{array}$ \\
"Vamos a las escuelas, vamos con los directores de las telesecundarias, comenzamos a hacer toda una labor" (EDV) \\
\hline
\end{tabular}

La tabla 2 resume parte de los elementos de la evaluación del proceso y del producto con base en nuestro esquema de análisis, es decir, las estrategias e intervenciones institucionales encaminadas al incremento de la cobertura. La efectividad de la tarea del área de vinculación se ve reflejada, en particular, en la creación de los TBC, que en Guanajuato se han multiplicado de manera considerable si los comparamos con el resto del país. En ello influye la labor de vinculación con los actores de las comunidades y los municipios, con los delegados y los directores en las telesecundarias, a fin de sondear que se pueda alcanzar la matrícula mínima necesaria para dar inicio con el servicio educativo.

Toda esta labor es, en gran medida, responsabilidad de los promotores que provienen de los CAE en cada municipio. La UVEG hace uso de sus recursos y aprovecha su presencia en todo el estado, de tal forma que, en realidad, no son nuevos actores, ya que llegan con el respaldo de la autoridad municipal, lo que facilita la gestión y vinculación inicial. 
Los responsables de los TBC justifican la creación de estos a partir de la urgencia de atender el rezago educativo, lo que, a su vez, les permite argumentar la necesidad de aprovechar los recursos disponibles, como las instalaciones de las telesecundarias. Ante la imposibilidad de tener infraestructura propia, le dan prioridad a "aprovechar los espacios que se dejan en telesecundaria porque no hay infraestructura, se prefiere ponderar el asunto de la docencia" (EDEM):

Anteriormente era el esquema de que el estudiante fuera a cabecera [del municipio] o a alguna comunidad más grande, donde había instalaciones educativas, y ahora no, el esquema es vamos a la comunidad con nuestros tres profesores a las instalaciones de telesecundaria, salvo a algunas excepciones donde nos hemos instalado en casas ejidales, en alguna primaria, porque la circunstancia, la infraestructura no era la idónea, había casos en que las escuelas estaban casi cayéndose o la telesecundaria estaba abandonada (EDEM).

El margen de acción, desde esta perspectiva, radica en los docentes; por lo tanto, su perfil, formación y desempeño tendrían que ser prioritarios por ser la influencia más evidente que se tiene, en la UVEG, para intervenir de forma clara en los TBC.

Ahora bien, parte del funcionamiento de los TBC y de su relativo arraigo y aceptación en las comunidades puede explicarse, en cierta medida, porque este modelo no solo aprovecha la infraestructura de las telesecundarias, sino también la historia de esos espacios, ya que en no pocos casos a los pobladores les ha costado darles vida y mantenerlas, de ahí que se entienda la participación de los padres de familia en la toma de decisiones, en la figura de los patronatos, en las gestiones y la aplicación de los recursos. Su participación, aunada al elemento comunitario y a la trayectoria de los estudiantes del TBC, no podría entenderse sin la misma historia de la telesecundaria, "porque hay que recordar que los hijos egresados de telesecundaria son los hijos egresados de telebachillerato [...] es la misma comunidad, es su misma escuela, por eso tienen un gran arraigo los estudiantes y los papás obviamente" (EDEM).

Sin embargo, la participación importante de las comunidades y su sentido de arraigo hacia las instituciones escolares no guarda alguna relación con el número creciente de TBC. Ello lo corroboran los comentarios de los actores entrevistados, quienes aseveran que ninguna gestión propia de las comunidades ha desembocado en la creación de un TBC: "No tengo conocimiento que haya sido así, se tramita a la Secretaría de Educación de Guanajuato, ellos hacen el estudio, la notificación de que requeriríamos un bachillerato en esa comunidad y nada más se notifica" (EDEM).

Los responsables del área de vinculación aseguran que la apertura de los numerosos TBC en el estado de Guanajuato se debe a las características propias de la entidad, con menor capacidad de cobertura en el nivel medio superior, en particular con comunidades y poblaciones que solo contaban con telesecundarias y no había más opciones educativas para que los jóvenes continuaran sus estudios:

¿Por qué abrimos tantos? Por eso, porque se veía la necesidad, porque los muchachos aparte de su comunidad, los que tenían posibilidad y eran pocos, podían salir a lo mejor a la cabecera municipal a recibir otro tipo de educación, pero estamos hablando de que tenían que invertir dinero en transporte, a veces en algo para que ellos pudieran permanecer en la cabecera, me refiero a comer o dormir y después regresar. Pero eran los menos, la verdad es que la pobreza que hay en las comunidades no les permitía que los niños salieran (EDAV). 
Sin duda, las condiciones de cobertura del estado y las necesidades de la población a la que van dirigidos los TBC dan razones suficientes para su apertura masiva. Sin embargo, los mismos responsables reconocen que este crecimiento exponencial -que "al principio fueron diez telebachilleratos, después ciento cuarenta y dos, después ciento sesenta y nueve. Ahorita estamos esperando los estudios de factibilidad para la apertura de otros, y no vamos por más de diez" (EDAV)-, se ha detenido y los ha llevado a plantear otro tipo de necesidades. Están en un momento en que buscan consolidar los ya existentes, pues pasan por un proceso de revisión de las áreas de oportunidad y fortalecimiento de lo ya instalado. Lo anterior ha sido provocado por lo que se percibe como un cambio en el tema de la cobertura en las zonas rurales; es decir, sostienen que han agotado todas las posibilidades de crecimiento, pues han llegado hasta donde podían hacerlo en las zonas rurales, al menos así lo han entendido en la UVEG:

... el tema cobertura de la burbuja poblacional que hoy tenemos ya no es un tema de comunidades, ya no es un tema rural, es un tema urbano, es un tema del corredor industrial, es un tema de León, Celaya, Irapuato. Y este modelo en particular, llega a las comunidades, llega a las zonas rurales, es por eso que [...] el último año creo que agotamos por completo las comunidades (EDAV).

El crecimiento de los TBC de 2013 a 2015 fue determinante: 10, 142 y 179, en ese orden, cubrieron casi en su totalidad lo que podían atender. En 2016 empezó a bajar la cifra, ya que solo se abrieron 20, y en 2017 esperaban tener 10 más. De ese modo, en tres años el TBC en Guanajuato, según los responsables, logró atender a las poblaciones que eran su objetivo; desde el inicio las matrículas han sido pequeñas y así continúan. Las posibilidades de los TBC dependen del comportamiento de la demanda en el nivel antecedente, es decir, en las telesecundarias; los mismos responsables aseguran que esta también ha mostrado un descenso. Si consideramos esta lógica, podemos inferir que la historia de los TBC puede ser corta.

\section{Problemáticas y necesidades de los TBC}

El tercer momento después de la gestión y creación es el funcionamiento, con el cual surgen las problemáticas que se van creando o irrumpiendo durante el proceso; una de ellas es el conflicto asociado al uso compartido de las instalaciones entre la telesecundaria durante la mañana y el TBC en la tarde. La perspectiva de los responsables del TBC en la UVEG nos permite observar parte de lo anterior; también el uso de los términos adelanta la manera en que conciben los TBC: "La negativa a lo mejor o la renuencia por parte de las personas que nos prestan las instalaciones, nosotros primero le damos la batuta al responsable del centro para que sea gestor de su propia unidad de negocio" (EAV).

En efecto, la búsqueda de acuerdos y soluciones recae en los encargados de la dirección de cada escuela, que son los mismos docentes que cumplen esa doble función. La intención es que exista un acuerdo entre quien representa la dirección de la telesecundaria con el equivalente del TBC, y que sea en ese nivel donde se den las soluciones referentes a la convivencia y el uso compartido de las instalaciones. De no existir en esa primera instancia una solución, se escala a los supervisores para lograr convenios en este aspecto. 
Los conflictos por el uso compartido de los espacios han sido de tal intensidad que han propiciado que la UVEG y la Secretaría de Educación del estado, en el marco del convenio vigente, lleguen a un pacto de sana convivencia para determinar algunos aspectos que tratan de revertir o gestionar problemáticas de esta naturaleza; por ejemplo, fijar con claridad la hora de entrada y salida, los salones que "se van a prestar", el uso del inmobiliario y su cuidado, así como la limpieza de las instalaciones. Ese acuerdo les permite, en particular a los docentes del TBC, tener una referencia a la cual apelar ante los variados conflictos que surgen por compartir un mismo espacio con las telesecundarias.

Al no existir inversión en infraestructura, se tiene que operar con lo que la telesecundaria dispone, o bien, con lo que le permiten usar; sin embargo, esta situación le abre un amplio margen al responsable de dirección de cada TBC para hacer gestiones pertinentes ante diferentes instancias. De conseguirse algún apoyo, al ser las instalaciones compartidas, debe existir además el consentimiento de la telesecundaria; una de las fuentes de apoyo a las que más se recurre y que suele funcionar es el municipio.

Otra de las problemáticas que enfrenta el TBC en el estado de Guanajuato ha sido, de entrada, la de su reconocimiento ante la postura de desconfianza de las comunidades. Más que la UVEG, como institución, han sido los promotores quienes han representado la avanzada en la vinculación con las autoridades y comunidades. En un principio, la desconfianza de los padres de familia radicaba en la validez, alimentada por la falta de instalaciones propias, que obliga a hacer uso en las tardes de las telesecundarias; esto generaba duda sobre su certeza y permanencia. Con el egreso de la primera generación, el cambio en la perspectiva de las comunidades ha sido evidente, aunado a la formalidad que ello implica, es decir, el ritual de la graduación y la entrega de papeles formales y oficiales. Los responsables del TBC se refieren a esta situación: "Es un proyecto de fe" (EDAV); esto, en el sentido de confiar en que se lograra el proceso educativo de nivel medio a pesar de que no se contaba con un establecimiento propio.

Uno de los elementos que más destaca es la ausencia de cuestiones propiamente pedagógicas. Los responsables de los TBC en la UVEG no abordan problemáticas de fondo de un proceso eminentemente educativo, porque, quizá, la instancia que tiene mayor peso es la del área de vinculación, cuyo foco y perspectiva no es la parte académica. Lo anterior se refuerza con los temas que son de interés y tratamiento en las áreas, por ejemplo, el reporte de incidencias, asuntos administrativos propios de control escolar, recursos humanos, finanzas, sistemas, etcétera.

En cambio, las problemáticas y necesidades identificadas por la Dirección de Educación Media debieran mostrar una perspectiva distinta; sin embargo, las dificultades en el TBC se relacionan con el ajuste o la alineación con las políticas en materia de EMS, en lo que antes era el Sistema Nacional de Bachillerato, ahora denominado Padrón de Buena Calidad; esto se traduce en el mero cumplimiento de una serie de requisitos y normas que les otorgan a las escuelas una suerte de certificación. El proceso y las dificultades implícitas en el TBC surgen en los comentarios de los entrevistados: "Nuestro esquema, nuestro modelo educativo [...] debemos entender que no son circunstancias de un plantel [...] son circunstancias excepcionales que se han atendido" (EDEM). 
Las condiciones excepcionales radican en lo que es el TBC y en cómo fue definido y concebido: en su plantilla docente básica o mínima, es decir, tres profesores, instalaciones prestadas, alejadas de centros urbanos, baja matrícula y escasos recursos pedagógicos, así como un perfil docente poco apegado al perfil deseable. En esas circunstancias es, en efecto, difícil cumplir con lo que se espera en el Padrón de Buena Calidad, por ejemplo, el tipo de planta docente, las actividades y funciones del director, las instalaciones y el equipamiento, entre otros aspectos.

Otro tipo de problema lo representan las trayectorias de los estudiantes, en particular en su permanencia y conclusión en tiempo y forma de sus estudios. Para los responsables, al igual que para los docentes y alumnos en las escuelas en las que hemos realizado nuestro estudio, "un problema quizás hasta social es que los chicos deciden casarse o insertarse de inmediato en la vida laboral, no tenemos tantos índices, no nos alarma demasiado, pero sí empezamos a ver la problemática de que los chicos empiezan a decidir si casarse o trabajar es la decisión para su edad" (EDEM).

\section{Impacto de los TBC en los jóvenes}

Los responsables administrativos y operativos de los TBC tienen claridad sobre el impacto del establecimiento de estos bachilleratos. Al respecto, los docentes expresan: "Hace tres años ellos ni siquiera pensaban en estudiar la preparatoria, pero ahorita, al abrirles los espacios, es muy muy bueno" (EAV). El efecto que los responsables destacan es el de brindar oportunidad de estudio a los jóvenes de las comunidades donde funcionan los TBC, cuyo resultado, según comentan en alusión al eslogan del gobierno estatal, es "un Guanajuato más educado" (EDV). Podríamos agregar a este lema el incremento de la cobertura y la posibilidad de que estudien los sectores de la población que no lo hacían por distintas razones, en particular los jóvenes de los medios rurales donde se localizan los TBC.

Asimismo, los responsables de los TBC mencionan que esta nueva opción educativa "no te lleva a necesariamente tener que insertarte en los corredores industriales, sino que te lleva a que tú puedas fortalecer a tu comunidad a través de los proyectos comunitarios" (EAV). El componente de desarrollo comunitario al que se le otorga un peso importante en el currículo del TBC cobra sentido como un elemento de distinción respecto a los demás subsistemas. En este aspecto, los responsables advierten el impacto de los TBC, ya que en la materia de desarrollo comunitario los estudiantes deben generar un proyecto "de emprendimiento" que apoye a la comunidad. Los logros en este rubro no son muy evidentes; sin embargo, ellos hablan de proyectos de algunos estudiantes y cuyos productos han vendido en los mercados y las ferias artesanales.

El cambio que perciben a largo plazo los responsables de los TBC proviene del progreso que históricamente se ha esperado de la educación: "Esto va a generar algo importante en el estado y yo lo veo como a diez años, las comunidades van a dejar de ser quienes eran para ser comunidades diferentes, porque creo que la educación cambia todo" (EAV). Lo anterior parece encontrar asideros en las mismas percepciones que los responsables de los TBC tienen sobre la manera en que conocieron a las comunidades y cómo, en su opinión, han venido transformándose: 
Cuando nosotros empezamos a abrir esos ciento cuarenta y dos [TBC] e íbamos a las comunidades, veíamos qué pasaba ahí y era muy común ver a los muchachos desde muy temprano tomando, porque no había otra cosa qué hacer, entonces en los dispensarios de cerveza o en los carros no haciendo nada, y hoy esa gente ya tiene algo qué hacer, ya tiene una etapa de continuidad, ya puede ir a la preparatoria y ya puede acceder al nivel educativo que le va ayudar a tener mejores condiciones para poder enfrentar la vida (EAV).

Esos cambios llevan a asumir a los actores responsables que el impacto no solo es a escala personal en los estudiantes, sino en las comunidades completas, aunque no se advierte en qué sentido o en qué aspectos las comunidades cambiarían. Podemos inferir que es en la conjunción del nivel educativo de los jóvenes y en el componente comunitario que parece tener prioridad en el currículo del TBC. El sentido de "salvación" subyace en los comentarios de los actores responsables del TBC, y permite cuestionar el impacto de este modelo en los jóvenes, más allá de las impresiones de los actores consultados.

A manera de hipótesis -y considerando que no presentamos en este texto los resultados del trabajo de campo con los docentes y los jóvenes-, el impacto tendría que mostrarse en la generación de los proyectos comunitarios y en la continuación de los estudios universitarios; sin embargo, lo anterior no parece estar sucediendo de manera significativa; apenas se tienen algunas referencias por parte de los responsables en la UVEG. Un análisis comparativo de lo que hacían los jóvenes antes del TBC y lo que hacen después de salir de este podría arrojar más luz para tener un acercamiento al impacto real que está representando para las poblaciones de estas comunidades el acceder a estudios de nivel medio superior.

La prospectiva, en términos del impacto, se orienta a las cuestiones académicas, aunque el área de vinculación, en teoría, no se encarga de este aspecto, pero ayuda a mostrar el traslape que hemos destacado. Así, el futuro que visualizan los responsables es la consolidación de los TBC ya creados y no establecer más: “... que desde los maestros, tengamos un personal calificado, capacitado porque han estado inmersos en varias capacitaciones tanto virtuales como desde la misma federación" (EAV).

\section{Un proyecto "noble"}

Tanto los responsables como los docentes de los TBC mencionan en forma reiterada que estos son un sistema o proyecto "noble". ¿En qué consiste esa nobleza? Para ellos, en los beneficios a las poblaciones desfavorecidas a las que se está brindando educación media:

Ha sido muy noble el proyecto lo tengo que mencionar, hemos tenido alrededor de catorce mil estudiantes en lo que llevamos, de 329 telebachilleratos en el estado de Guanajuato, ya estamos en los 46 municipios, ya estamos casi en el último rincón del estado ahí hay un telebachillerato [...]. Creo que es un proyecto noble como le había mencionado, tiene un gran impacto social, estamos cambiando para bien, más en la gente, incluso en lo ideológico, gente que pensaba que más allá de su comunidad no iba a tener desarrollo o gente que pensaba que solo emigrando a los Estados Unidos iban a tener desarrollo, créame que ha sido uno de los grandes retos, quitar esa ideología de "me quiero ir para el Norte" porque ahí es donde está el futuro (EDEM).

Ese sería uno de los impactos que los responsables de los TBC consideran que estos tienen en los jóvenes de las comunidades a las que llegan. Como parte del mismo 
proceso, destacan la ventaja del trato personalizado, pues son grupos pequeños con los que opera cada TBC; aunque comentan que hay algunas escuelas que tienen más de cien estudiantes, la amplia mayoría lo hace con matrícula de entre 15 y 25 jóvenes. Esto lo califican como un plus, un signo de distinción en la formación de los estudiantes, ya que abona a un tratamiento personalizado que genera confianza y cercanía entre docentes y jóvenes.

\section{CONCLUSIONES}

La caracterización de los TBC se generó mediante la revisión de sus principales documentos y del trabajo de campo con los responsables en el estado de Guanajuato. El modelo seguido en nuestro estudio busca destacar los tres propósitos evaluativos que lo integran: servir de guía para la toma de decisiones, aportar datos para la responsabilidad, y promover la comprensión de los fenómenos implicados (Stufflebeam y Shinkfield, 1987).

A diferencia de otros modelos, este ha resultado pertinente, pues está dirigido a promover el desarrollo y, en particular, a ayudar a directivos y responsables a obtener y utilizar información para satisfacer las necesidades de estos actores. Sin embargo, aquí también puede residir su debilidad si se buscan otros propósitos, como atender de manera preponderante la perspectiva de los actores usuarios, que en este caso son los jóvenes estudiantes, las familias y las comunidades donde se establecen los TBC.

En virtud de que nuestro interés se centra en la caracterización de los TBC con base en sus documentos principales y en el trabajo de campo con los responsables estatales, y ajustándonos a cada momento del método CIPP, concluimos lo siguiente:

- En la evaluación del contexto, puede parecer lógico que la operación de los TBC haya sido asignada a la UVEG por su experiencia en lo virtual y porque ha implementado estrategias de ese tipo para mantener comunicación con las escuelas; no obstante, los TBC no funcionan de manera virtual, sino presencial. La ventaja más bien radica en la presencia que en cada municipio tiene la UVEG a través de los CAE. Es una fortaleza que lograron aprovechar como su avanzada en las comunidades y que facilitó, en buena medida, la identificación de las localidades, y el acuerdo y seguimiento para la instalación de los TBC. En la figura de los CAE puede encontrarse parte de la explicación del crecimiento exponencial en el número de TBC en Guanajuato.

Por sus características, empezando por el nombre, podría pensarse que el ámbito de lo comunitario habría implicado una participación decisiva de las comunidades en las escuelas desde su gestación. Sin embargo, lo mostrado aquí revela que han sido más bien receptoras de las gestiones iniciadas en otras áreas y ámbitos de decisión. Incluso, a las comunidades se les ha tenido que convencer para que apoyen a las escuelas enviando a sus hijos. Quizá por el tipo de gestión inicial resultó difícil al principio que las comunidades se apropiaran, en el mejor sentido, de las escuelas, y solo el tiempo y los resultados han cultivado la confianza hacia los TBC. 
- En la evaluación de entrada se hizo evidente que el área de vinculación tiene mayor peso y presencia en las escuelas. Sin embargo, no atiende lo pedagógico; da seguimiento a los procesos formales de gestión y funcionamiento. De ahí que mantenga un discurso oficial y con poca crítica hacia los resultados del TBC. Sin duda, cumple una función importante, pero frente a su interés por la consolidación de lo logrado. Por ello, tendría que encauzar más su apoyo hacia la mejora de los procesos de enseñanza-aprendizaje. El sistema que ha implementado para la comunicación con las escuelas, que privilegia los procesos de gestión y control escolar, bien podría aprovecharse para brindar apoyo académico a los docentes.

Reconocemos que el margen de acción que se tiene desde la UVEG hacia los TBC es limitado en distintas áreas, por ejemplo, en infraestructura. Su ámbito de acción recae sobre todo en los docentes, en lo referente a su contratación, formación y desempeño, así como en retomar los resultados de las evaluaciones y los logros educativos de los TBC para emprender procesos de mejora. Sin embargo, estos aspectos están poco desarrollados y aprovechados, a pesar de la pretensión de consolidar lo logrado.

La expresión distintiva de los TBC (Bourdieu, 1988) es la materia de desarrollo comunitario; en realidad, esa es la diferencia que los responsables ven en esta opción educativa respecto a los demás subsistemas de educación media. Aunque aún sin impactos evidentes, sería una apuesta para el momento de consolidación que los responsables dicen estar iniciando, al igual que la formación docente, que puede ser su ámbito de intervención más claro.

- En la evaluación del proceso, es importante señalar que la innovación curricular que representa el TBC se ha topado con el perfil docente y las condiciones de operación y lógica con base en las cuales fueron creados; es decir, planta docente mínima, perfiles docentes no apropiados para cumplir con lo determinado en la RIEMS, escasos apoyos pedagógicos en campo, entre otros. Trabajar, por ejemplo, con proyectos formativos sigue siendo un propósito bien planteado en la propuesta curricular, pero en el contexto real no se ha dispuesto su funcionamiento. Lo mismo sucede con la materia de desarrollo comunitario; las expectativas y el peso han sido altos, pero, al igual que lo ha mostrado por Weiss (2017), en la práctica no se han visto los impactos.

El proceso de creación del TBC muestra que, para la apertura de un centro nuevo, lo que se requiere básicamente es el cumplimiento de una cuestión administrativa: verificar determinados requisitos, pero el involucramiento sustantivo de la comunidad no parece claro en un inicio. Si las comunidades, aunque con recelo al principio, han terminado incorporando a los TBC a su dinámica, ha sido por la historia misma de la telesecundaria, institución importante en ellas y estratégica para la existencia de los TBC; esto, a pesar de que se han sentido invadidas en sus espacios. Lo cierto es que los TBC no pueden entenderse sin las telesecundarias; más aún, si no se refuerza el trabajo de estas en cuanto a la mejora de instalaciones, cobertura y calidad, el costo se transferirá de manera irremediable al TBC.

- La evaluación del producto revela que la intención de que esta modalidad propicie la continuación de estudios no es prioritaria y, por ello, es 
poco fomentada. El impacto se resuelve en la perspectiva del apoyo y la mejora de la comunidad a través de su signo de distinción: la materia de desarrollo comunitario.

En su planeación y estructura, en el currículo, el TBC resulta relevante y pertinente por estar adecuado al ámbito de la comunidad y a las expectativas y circunstancias de vida de los estudiantes (INEE, 2007, 2009). La formación para el trabajo, como es considerada la materia de desarrollo comunitario, gira en torno al desarrollo de proyectos en beneficio de la comunidad y de los jóvenes estudiantes. No obstante, la insuficiencia hasta ahora resulta ser el desarrollo e impacto claro de dicha formación, que marca, como hemos aseverado, una diferencia respecto a todos los demás subsistemas de educación media, y para ser tal, es decir, su valor agregado, debería ser efectiva en cada escuela y en cada proyecto.

La equidad, entonces, en este subsistema se refiere claramente a brindar educación media a sectores de la población excluidos de la posibilidad de estudiar, o bien, que les representaba un gasto que los ponía en constante riesgo de abandonar la escuela. La pregunta es si esta igualdad en cantidad (Formichella, 2011), es decir, en cobertura, y no en calidad en aprendizajes y continuación de los estudios, basta para afirmar que el TBC es una respuesta de equidad del Estado mexicano hacia este sector de la población.

\section{REFERENCIAS BIBLIOGRÁFICAS}

Álvarez-Gayou, J. (2003). Cómo hacer investigación cualitativa. Fundamentos y metodología. México: Paidós.

Angrosino, M. (2012). Etnografía y observación participante en investigación cualitativa. España: Morata.

Bourdieu, P. (1988). La distinción. Criterios y bases sociales del gusto. España: Taurus.

De la Orden, A. (2007). Evaluación de la calidad de la educación. Un modelo sistémico como base para la construcción de un sistema de indicadores. En INEE. Seminario Internacional de Indicadores Educativos. Conceptos, metodologías y experiencias para la construcción de un sistema de indicadores educativos (pp. 6-21). México: INEE.

Diario Oficial de la Federación (2009). Acuerdo 444 por el que se establecen las competencias que constituyen el Marco Curricular Común del Sistema Nacional de Bachillerato. México. Recuperado de http://www.sems.gob.mx/work/ models/sems/Resource/10905/1/images/Acuerdo_444_marco_curricular_ comun_SNB.pdf

Diario Oficial de la Federación (2008). Acuerdo 447 por el que se establecen las competencias docentes para quienes impartan educación media superior en la modalidad escolarizada. México. Recuperado de http://www.sems.gob. $\mathrm{mx} /$ work/models/sems/Resource/11435/1/images/5_4_acuerdo_447_ competencias_docentes_ems.pdf

Espinoza, E. (2014). Dificultades en lengua escrita en español de estudiantes de telebachillerato de contexto otomí. Tesis de maestría en Investigación Educativa, Universidad Veracruzana, México. 
Formichella, M. (2011). Análisis del concepto de equidad educativa a la luz del enfoque de las capacidades de Amartya Sen. Revista Educación, vol. 35, núm. 1, pp. 1-36. Recuperado de https://revistas.ucr.ac.cr/index.php/educacion/ article/view/25150

INEE (2017). Panorama educativo de México 2016. Indicadores del sistema educativo nacional. Educación básica y media superior. México.

INEE (2016). Panorama educativo de México 2015. Indicadores del sistema educativo nacional. Educación básica y media superior. México.

INEE (2015). Panorama educativo de México 2014. Indicadores del sistema educativo nacional. Educación básica y media superior. México.

INEE (2014). Panorama educativo de México 2013. Indicadores del sistema educativo nacional. Educación básica y media superior. México.

INEE (2013). Panorama educativo de México 2012. Indicadores del sistema educativo nacional. Educación básica y media superior. México.

INEE (2012). Panorama educativo de México 2011. Indicadores del sistema educativo nacional. Educación básica y media superior. México.

INEE (2009). Panorama educativo de México. Indicadores del sistema educativo nacional. México.

INEE (2007). Propuesta y experiencia para desarrollar un sistema nacional de indicadores. México.

Omaña, 0. (2001). El telebachillerato, una forma de utilizar la televisión con fines educativos. Comunicar, núm. 16, pp. 171-176. Recuperado de https:// www.revistacomunicar.com/index.php?contenido=detalles\&numero=16\&ar ticulo=16-2001-23

Ramírez, R. (coord.) (2015). Los desafíos de la educación media superior. México: Senado de la República, Instituto Belisario Domínguez.

Rosas, M., Santiago, M. y Lara, R. (2015). Educación y reproducción de sociedades no capitalistas. El caso de alumnos de telebachillerato y la Empresa Forestal Comunitaria de San Pedro el Alto, México. Perfiles Educativos, vol. XXXVII, núm. 149, pp. 94-111. Recuperado de http://www.iisue.unam.mx/perfiles/ articulo/2015-149-educacion-y-reproduccion-de-sociedades-no-capitalistas-el-caso-de-alumnos-de-telebachillerato-y-la-empresa-forestal.pdf

Salazar, P. (2007). Un estudio de estrategias y prácticas de los docentes en relación con las matemáticas de los profesores del telebachillerato en el estado de Veracruz. Tesis de maestría en Matemática Educativa, IPN, México.

SEMS (2018). Evolución del subsistema de telebachillerato. Recuperado de http://www.sems.gob.mx/telebachilleratos

SEMS (2017). Mapa curricular del bachillerato general para el telebachillerato comunitario. Componente de formación para el trabajo en desarrollo comunitario. México: SEP-SEMS.

SEMS (2016). Documento base. Telebachillerato comunitario. México: SEP.

SEMS (2014). Documento base para el servicio educativo de telebachillerato comunitario. México: SEP.

SEMS (2014a). Desarrollo comunitario. México: SEP-SEMS, serie Programas de Estudio. 
SEMS (2014b). Mapa curricular del bachillerato general para el telebachillerato comunitario con un enfoque educativo basado en el desarrollo de competencias. México: SEP-SEMS.

Solís, P. (2018). La transición de la secundaria a la educación media superior en México: el difícil camino a la cobertura universal. Perfiles Educativos, vol. XL, núm. 159, pp. 66-89. Recuperado de http://www.iisue.unam.mx/perfiles/ articulo/2018-159-la-transicion-de-la-secundaria-a-la-educacion-media-superior-en-mexico-el-dificil-camino-a-la-cobertura-universal.pdf

Strauss, A. y Corbin, J. (2002). Bases de la investigación cualitativa. Técnicas y procedimientos para desarrollar la teoría fundamentada. Colombia: Universidad de Antioquia.

Stufflebeam, D. y Shinkfield, A. (1987). Evaluación sistemática. Guía teórica y práctica. Barcelona: Paidós/MEC.

Weiss, E. (2017). Estudio exploratorio del modelo de telebachillerato comunitario y su operación en los estados. México: INEE.

Weiss, E. (2017a). El telebachillerato comunitario. Una innovación curricular a discusión. Revista Latinoamericana de Estudios Educativos, vol. XLVII, núm. 3-4, pp. 7-26. Recuperado de http://www.redalyc.org/articulo. oa?id=27054113002

Weiss, E. (2015). El abandono escolar en la educación media superior: dimensiones, causas y políticas para abatirlo. En R. Ramírez (coord.). Los desafíos de la educación media superior (pp. 81-159). México: Senado de la República, Instituto Belisario Domínguez. 